\title{
NEUTRON SPIN STRUCTURE IN THE RESONANCE REGION AND QUARK-HADRON DUALITY
}

\author{
P. SOLVIGNON \\ FOR THE JEFFERSON LAB HALL A COLLABORATION \\ 12000 Jefferson Avenue \\ Newport News, VA 23606, USA \\ E-mail: solvigno@jlab.org
}

\begin{abstract}
The Thomas Jefferson National Accelerator Facility experiment E01-012 measured the ${ }^{3} \mathrm{He}$ spin structure functions and virtual photon asymmetries in the resonance region in the range $1.0<\mathrm{Q}^{2}<4.0(\mathrm{GeV} / \mathrm{c})^{2}$. Our data combined with existing deep inelastic data can be used to test quark-hadron duality on $g_{1}$ and $\mathrm{A}_{1}$ for ${ }^{3} \mathrm{He}$ and the neutron. The demonstration of duality for spin structure functions will enable the use of resonance data to study nucleon spin structure in the very high $x_{b j}$ region. Preliminary results of $\mathrm{A}_{1}^{3} \mathrm{He}$ will be presented as well as an overview of the experiment and theoretical developments.
\end{abstract}

\section{Introduction}

In the 70's, Bloom and Gilman ${ }^{1}$ observed that the nucleon resonances average on the high $\mathrm{Q}^{2}$ scaling curve when an appropriate scaling variable is used. Since then Bloom-Gilman duality has been experimentally demonstrated for the spin independent structure function $\mathrm{F}_{2}$ of the proton and the deuteron ${ }^{2}$ and for the virtual photon asymmetry $\mathrm{A}_{1}$ of the proton ${ }^{3}$.

Substantial efforts are ongoing in investigating quark-hadron duality in polarized structure functions both experimentally and theoretically. Carlson and Mukhopadhyay ${ }^{4}$ showed using perturbative QCD that the structure functions in the resonance region fall with increasing $\mathrm{Q}^{2}$ at the same rate as in the deep inelastic region. The behavior of $g_{1}$ in the resonance region at high $\mathrm{Q}^{2}$ is proportional to the helicity amplitude $G_{+}=g_{+} / Q^{3}$ and can be written as follow:

$$
g_{1}=\frac{m_{N}^{2}}{\pi m_{R} \Sigma_{R}} G_{+}^{2}=\frac{m_{N}^{2}}{\pi m_{R} \Sigma_{R}} \frac{g_{+}^{2}}{\left(m_{R}^{2}-M_{N}^{2}\right)^{3}}(1-x)^{3}
$$


where $g_{+}$is a constant and $1 / Q^{2} \approx \frac{1-x}{m_{R}^{2}-M_{N}^{2}}$ for $X \rightarrow 1$ and $W \approx M_{R}$. In the deep inelastic region, the photon is more likely to interact with the quark having the same helicity as the nucleon. This implies that $g_{1}$ and $F_{1}$ behave the same way as $\mathrm{x}$ approaches 1 and:

$$
g_{1}(x) \propto(1-x)^{3} \text { as } \mathrm{x} \rightarrow 1
$$

Finally they predicted $A_{1}$ tends to 1 as $x \rightarrow 1$ in the scaling region and the same is true for $A_{1}$ in the resonance region at high enough $Q^{2}$ considering resonant and non-resonant background.

Recently, Close and Melnitchouk ${ }^{5}$ studied three different conditions of $\mathrm{SU}(6)$ breaking applied in the resonance region under which predictions of the structure functions at large $\mathrm{x}$ lead to the same result as the parton model. They examined the cases where certain resonances are removed from the summation, (that is, suppression of spin- $\frac{3}{2}$, suppression of helicity- $\frac{3}{2}$ and suppression of symmetric wave function), and found that each scenario predicts $\mathrm{A}_{1}^{n, p} \rightarrow 1$ as $\mathrm{x} \rightarrow 1$.

Now that precise spin structure data in the deep inelastic region ${ }^{6}$ are available, data in the resonance region is needed (especially for the neutron) in order to test duality in the polarized case. Thus the goal of the experiment E01-012 was to produce such data in the moderate $\mathrm{Q}^{2}$ region of 1.0 to $4.0(\mathrm{GeV} / \mathrm{c})^{2}$ where duality is expected to hold.

\section{The E01-012 experiment}

E01-012 ran successfully in January-February 2003 at Jefferson Lab in Hall A (see fig. 1). It was an inclusive experiment of longitudinally polarized electrons scattering on a longitudinally or transversely polarized ${ }^{3} \mathrm{He}$ target ${ }^{7}$. Asymmetries and cross section differences are formed in order to extract the spin structure function $g_{1}$ and the virtual photon asymmetry $A_{1}$ in the resonance region up to $Q^{2}=4(\mathrm{GeV} / \mathrm{c})^{2}$ :

$$
\begin{gathered}
g_{1}=\frac{M Q^{2} \nu}{4 \alpha_{e}^{2}} \frac{E}{E^{\prime}} \frac{1}{E+E^{\prime}}\left[\Delta \sigma_{\|}+\tan \left(\frac{\theta}{2}\right) \Delta \sigma_{\perp}\right] \\
A_{1}=\frac{A_{\|}}{D(1+\eta \xi)}-\frac{\eta A_{\perp}}{d(1+\eta \xi)}
\end{gathered}
$$

where $A_{\|}\left(A_{\perp}\right)$ is the parallel (perpendicular) asymmetry corrected for data 
acquisition deadtime, beam charge asymmetry, target and beam polarizations and nitrogen dilution. $\Delta \sigma_{\|(\perp)}=2 A_{\|(\perp)} \sigma_{0}$ with $\sigma_{0}$ the unpolarized cross section, and $\eta, \xi, \mathrm{D}$ and $\mathrm{d}$ are kinematic factors (see for example ${ }^{6}$ ). To determine $\mathrm{D}$ and $\mathrm{d}$, world data for $R\left(x, Q^{2}\right)$ will be used. However our data allows a direct extraction of $g_{1}$ and $g_{2}$ without the need of external input.

The beam polarization measured using a Moller polarimeter was on average $77(1 \pm 0.03) \%$. The polarization of the target was determined by two independents polarimetries ${ }^{7}$ : NMR and EPR. From the preliminary analysis, the target polarization was $37(1 \pm 0.04) \%$ on average. Two almost identical spectrometers were used in a symmetric configuration in order to double our statistics and check our systematics. In order to select good scattered electrons, a gas cerenkov counter along with a two-layer electromagnetic calorimeter was used in the analysis. These allowed us to reduce the pion contamination by a factor better than $10^{4}$ while keeping the electron efficiency above $99 \%$.

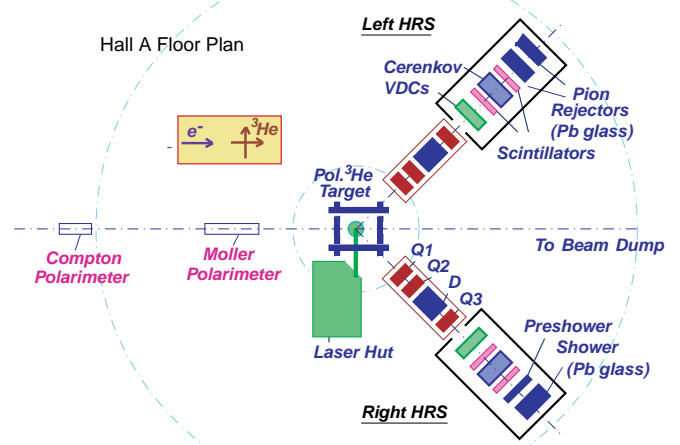

Figure 1. Hall A floor plan. The electron beam is coming from the left.

\section{Preliminary results}

For our first pass analysis, $\mathrm{A}_{1}^{3} \mathrm{He}$ was extracted from the asymmetries. $\mathrm{R}\left(\mathrm{x}, \mathrm{Q}^{2}\right)$ was assumed constant with a value of 0.18 . No radiative corrections are applied and the error bars are statistical only. Figure 2 shows $\mathrm{A}_{1}^{3} \mathrm{He}$ at four different $\mathrm{Q}^{2}$ values in function of the Nachtmann scaling vari- 
able $\xi=2 x /\left(1+\sqrt{1+\frac{4 M^{2} x^{2}}{Q^{2}}}\right)$. The position of the $\Delta(1232)$ resonance is indicated in each plot.

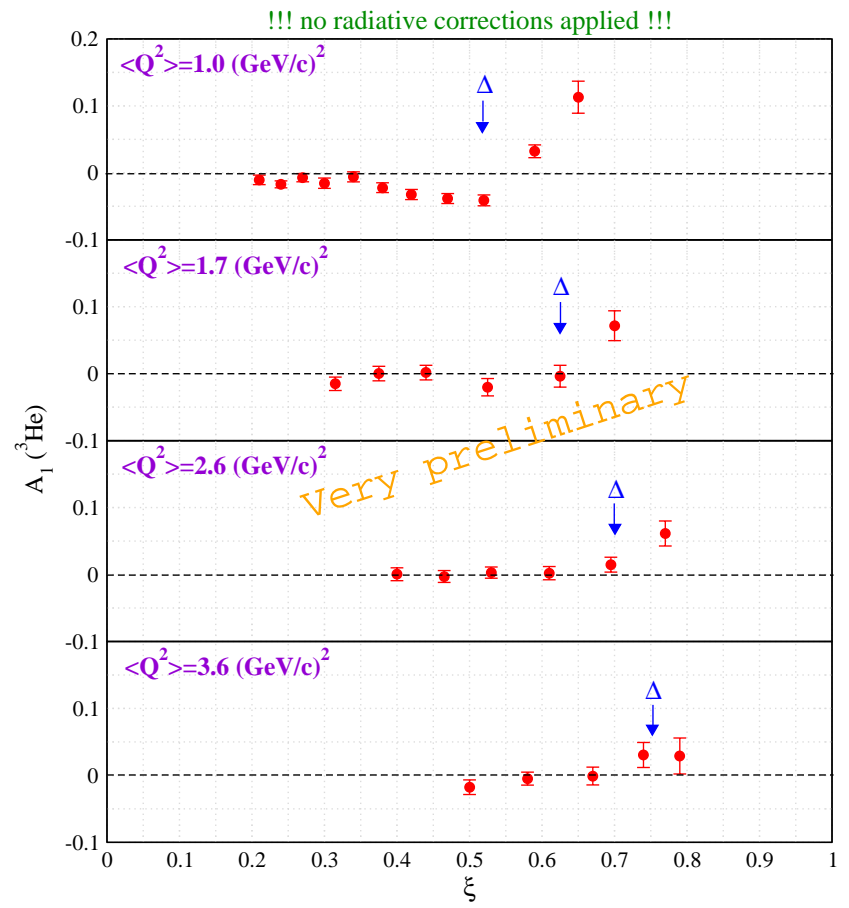

Figure 2. Preliminary result of $\mathrm{A}_{1}^{3} \mathrm{He}$. See text for discussions.

The most important feature is the negative contribution of the $\Delta(1232)$ resonance at low $\mathrm{Q}^{2}$. It is reported ${ }^{4,5}$ that quark-hadron duality is not expected to apply in the $\Delta$ region at this low $\mathrm{Q}^{2}$. However at higher $\mathrm{Q}^{2}$, $\mathrm{A}_{1}^{3} \mathrm{He}$ in the $\Delta(1232)$ increases due to the fall off of the resonance and the rising background. At large $\xi$, our data tend to follow the same pattern as the DIS world data ${ }^{8}$ and indicate the validity of duality.

\section{Summary}

E01-012 resonance data cover the region of $0.2<x<0.90$ (see fig. 3). At $\mathrm{x}<0.60$ where DIS data are available, we will provide a precision test of quark-hadron duality predictions for neutron spin structure functions. Morever, if duality is confirmed, E01-012 will provide the first precise measure- 


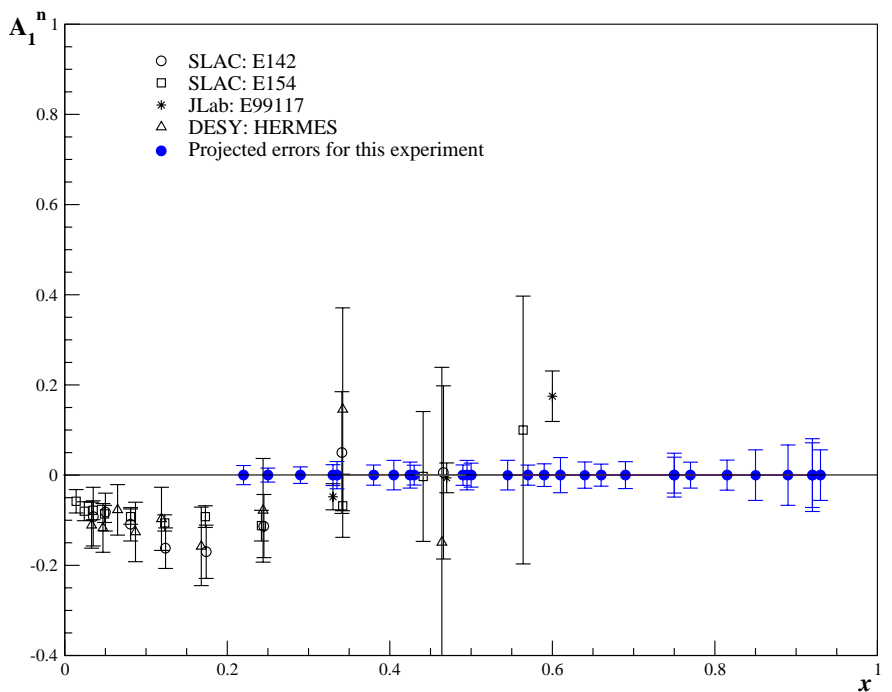

Figure 3. $\quad A_{1}^{n}$ results measured in the deep inelastic region using polarized ${ }^{3} \mathrm{He}$ targets and E01-012 projected statistical errors are shown.

ment of $\mathrm{g}_{1}^{n}$ and $\mathrm{A}_{1}^{n}$ in the range $0.60<\mathrm{x}<0.90$.

Our data will also be used to extract moments of the structure functions, for example: the extended GDH sum, $\mathrm{d}_{2}$ matrix element and the Burkhardt-Cottingham sum rule.

\section{Acknowledgments}

This work was supported by DOE contract DE-AC05-84ER40150 under which the Southeastern Universities Research Association (SURA) operates the Thomas Jefferson National Accelerator Facility.

\section{References}

1. E. D. Bloom and F. J. Gilman, Phys. Rev. Lett. 25, 1140 (1970).

2. I. Niculescu et al., Phys. Rev. Lett. 85, 1182 (2000); 85, 1186 (2000).

3. A. Airapetian et al., Phys. Rev. Lett. 90, 092002 (2003).

4. C. E. Carlson and N. C. Mukhopadhyay, Phys. Rev. D 58, 094029 (1998).

5. F. E. Close and W. Melnitchouk, Phys. Rev. C 68, 035210 (2003).

6. X. Zheng et al., Phys. Rev. Lett. 92, 012004 (2004).

7. K. Slifer, in Proceedings of the $2^{\text {nd }}$ International Symposium on the GDH Sum Rule and Spin Structure of the Nucleon, 1st ed., edited by M. Anghinolfi, M. Battaglieri and R. De Vita (World Scientific, New Jersey, 2003).

8. X. Zheng et al., to be published in Phys. Rev. C. 